\title{
Image Processing Based Fault Detection and Isolation for Mechanical Components
}

\author{
C. Bharathi Priya, V.Sudha
}

\begin{abstract}
Fault Detection and Isolation (FDI) is essential in mechanical industry to detect and isolate objects with manufacturing defect. At present in assembly line, mechanical components are transported from one stage to other stage for assembly, packing etc. During this process, components are randomly drawn from the conveyor belt and manually inspected. Since the random inspection is done manually, there is a chance of missing out defected components in the assembly line. Manual inspection is time consuming and all the features of the components cannot be verified accurately. Hence, there is a need for a image processing based system to detect the anomalies in the components sent in the conveyor belt. In this work, camera is mounted above the conveyor module and captures the images of nuts and bolts which moving on conveyor belt. Captured images are preprocessed to remove background noise, then image is enhanced to get the appropriate features and Region of Interest (diameter of nut) is extracted to measure the diameter. If any anomaly is found in the attributes (diameter) of the mechanical components, an electrical signal will be sent to the Solenoid valve and then it actuates deflector plate by the pneumatic cylinder. Defected component is then carried by the secondary conveyor to the re-matching and the quality product are then carried to the packaging will passed to the separator through microcontroller. In this way, components with manufacturing defect are identified and isolated from assembly line.
\end{abstract}

Keywords: Fault detection, Isolation, Segmentation, Feature Extraction.

\section{INTRODUCTION}

The main objective of automating the fault detection and isolation assembly line of mechanical industry is to reduce human efforts and error. Automating process of fault detection and isolation will more preciously detect the defected components, which would have been missed out by the human. Then a decision can be made for rejecting or accepting the components is taken by measuring quality parameters.

The fastener is a hardware device that mechanically joins two or more objects together. Bolts and Nuts are the example of fasteners used in mechanical /automobile industry. It is very much important to verify the parameters of fasteners before supplying it to industry. Normally these fasteners are inspected manually, which is time consuming and there is a chance for human error to miss out the faulty components. Hence there is a need for an image processingbased solution which identifies the faulty components moving in conveyor belt and isolate to a secondary conveyor belt.

Revised Manuscript Received on August 14, 2019.

C. Bharathi Priya, Assistant Professor II, Department of Computer Science and Engineering, Kumaraguru College of Technology Coimbatore. T.N, India.

V.Sudha, Assistant Professor II, Department of Computer Science and Engineering, Kumaraguru College of Technology Coimbatore. T.N, India
To measure quality parameters such as dimensions and features of the product is feasible by use of image processing techniques. Image processing techniques can be used to extract different properties of the objects such as their dimensions, areas, etc.

In this paper, section I deals with introduction, Section II reviews the few existing work, proposed system is explained in section III. Implementation details and results are discussed in section IV. In section V, summary of the work is discussed.

\section{LITERATURE SURVEY}

Computer Vision Based Object Sorting \& Fault Detection Using Ann [1] deals with identification of bolt and nut in real time for various application in industries. The objective of this work is to develop the image processing algorithm using back propagation to get the normalized cropping images which would be suitable for processing and detection.

Stationary wavelet transform is a technique used for the application for sorting of nuts and bolts on a conveyor belt in an industry. The optimization algorithm has few iterations than Artificial Neural Network process for the same task. Other improved algorithms have the convergence rate faster and the precision is higher.

Object Sorting Robot Using Image Processing [2] deals with Object Sorting by applying image processing techniques to detect and identify the colors. This robot has robotic arm has servo motor is used to pick and place the components and robotic arm would move in the vertical direction

The robot system consists of a visual sensor system used in the domain of robotics for object identification and object tracking. PC based camera is mounted which transfers the image to the program for processing and segmentation algorithm is applied for object. Once the object is being detected, robot arm is activated to track the object using serial COM port of the computer. Working of this system is divided into four phases: Image acquisition Segmentation, Feature extraction and motion control.

Fault Detection [3] using Image Processing approach proposed an approach to recognize fabric defects in textile industry for reducing production cost and time. Image analysis is performed on Fabric materials to detect the defect in the clothes. Image acquisition system acquires the image of the cloth and sends the image to recognizer. It converts that image into binary image by restoration and threshold techniques. This approach reduces the manual work and 
conserves time and produces quality material. Fault detection is completely dependent on the quality of the image acquired.

\section{PROPOSED SYSTEM}

The Basic principle of operation is starts with the object detection when the conveyor starts to transport the object. And the Camera is positioned in such a way to capture the image and sends to the computer for the dimensional extraction and the computer checks for the limits. If it is found that the component doesn't satisfy, then the computer sends the signal to the Solenoid valve and then it actuates deflector plate by the pneumatic cylinder and the defected component is then carried by the secondary conveyor to the re-machining and the quality product are then carried to the packaging. Fig 1 depicts the hardware setup constructed for the given problem statement.

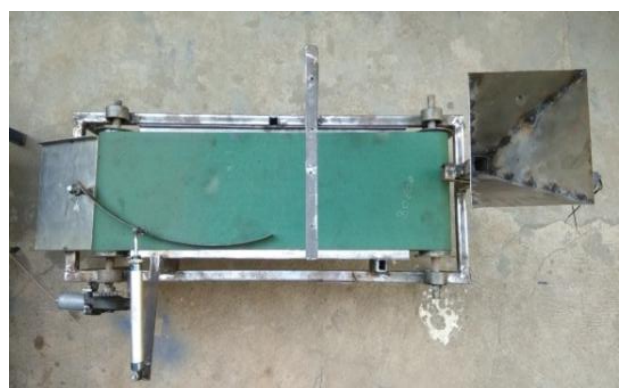

Fig 1 Hardware Setup

Image processing based fault detection and isolation is proposed to detect the anomalies in the fastener (Mechanical components. nuts/bolts) sent in the conveyor belt. In this work, camera is mounted above the conveyor module and captures the images of nuts and bolts which moving on conveyor belt.

Captured images are preprocessed to remove background noise, then image is enhanced to get the appropriate features and Region of Interest (diameter of nut) is extracted to measure the diameter[11]. If any anomaly is found in the attributes (diameter) of the mechanical components, an electrical signal will be sent to the Solenoid valve and then it actuates deflector plate by the pneumatic cylinder. Defected component is then carried by the secondary conveyor to the re-machining and the quality product are then carried to the packaging will passed to the separator through microcontroller. In this way, components with manufacturing defect are identified and isolated from assembly line.

\section{PHASES OF PROPOSED SYSTEM \& RESULTS}

The phases of the proposed system is depicted in Fig 2

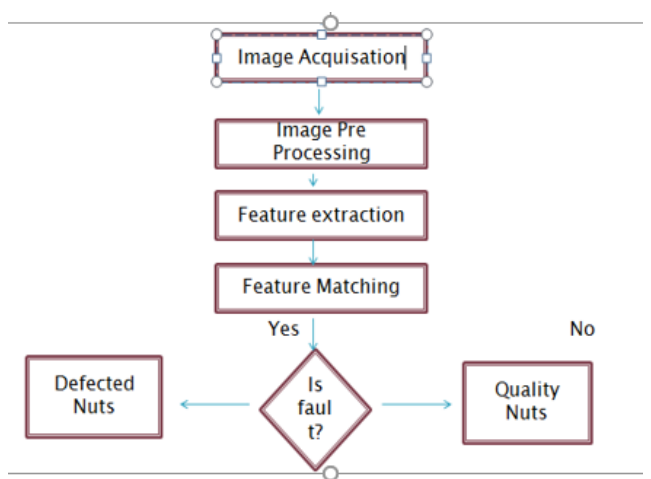

Fig 2: Phases of the proposed system

\section{Image Acquisition:}

In this system the nuts and bolts were moving on the conveyor belt. The camera which is on the conveyor belt setup will capture the images of nuts and bolts. Here we use CMOS Camera to capture the image in resolution of $320 \times 240$ pixels. Image acquisition is the first and most important step to capture the image. Hardware equipment carries a very important role to acquire image with sufficient contrast and sharp focusing. Each individual image was stored and further analyzing can be carried on thereafter.

\section{Image Preprocessing:}

In Image preprocessing, there are 2 main phases must be done. First is edge detection, Edge detection is an image processing technique for finding the boundaries of objects within images. It works by detecting discontinuities in brightness. Canny edge detection algorithm is used here for detect the edges of the object in the image. Second phase is image segmentation, image segmentation is the process of partitioning a digital image into multiple segments (i.e) sets of pixels, also known as super-pixels. The goal of segmentation is to simplify and/or change the representation of an image into something that is more meaningful and easier to analyze. Fuzzy segmentation algorithm is used to segment the object from the image. Image segmentation is typically used to locate objects and boundaries (lines, curves, etc.) in images.

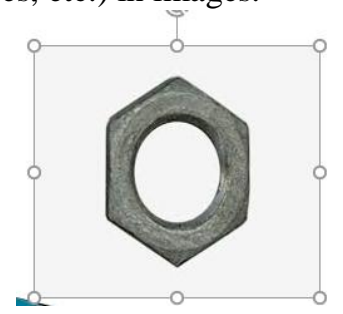

Fig 3 a. Input image

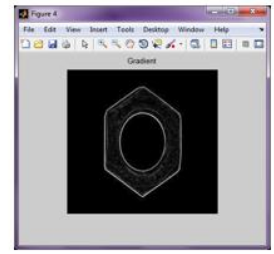

b. Edge Detection

\section{Feature Extraction}

We have $\mathrm{n}$ nuts and $\mathrm{n}$ bolts. The nuts (and the bolts) are of varied sizes. Each bolt fits in exactly 1 nut. We would like to match the nuts with the bolts which fits into them. Since the dimensions of the nuts and the bolts are so small, we can not really tell if a nut (or a bolt) is bigger than another nut (or bolt). So the only operation that is allowed is comparing a nut and a bolt - with such a comparison we can distinguish between three cases, namely, the nut fits a larger bolt, or the nut fits a 
smaller bolt, or the nut fits this bolt. For this comparison here we use Mathematical morphology algorithm to extract the feature of the object in the Image. Give a randomized algorithm which matches nuts with bolts. The expected number of comparisons (of a nut with a bolt) done by this algorithm should be $\mathrm{O}(\mathrm{n} \log n)$.

\section{Feature matching:}

From the extracted feature (inner circle) of the nut from the image, diameter is computed. If the diameter is greater or lesser than the predefined diameter, a signal is sent to embedded system

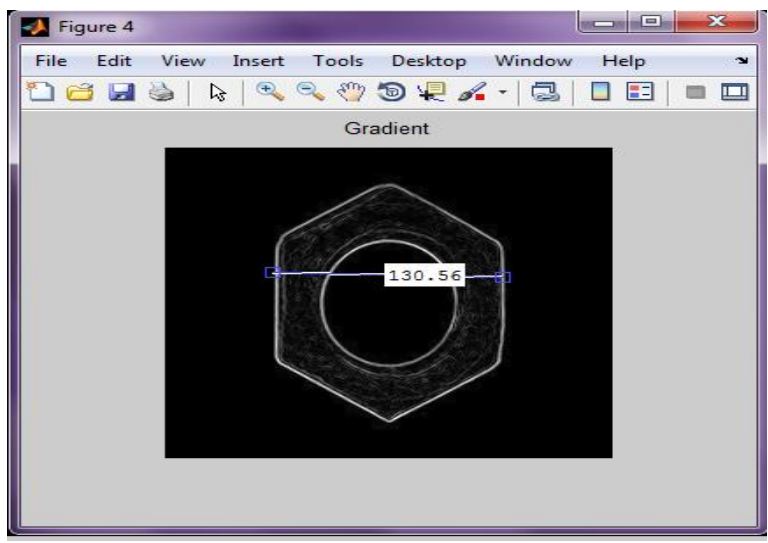

Fig 4 Feature Extraction

\section{IMPLEMENTATION}

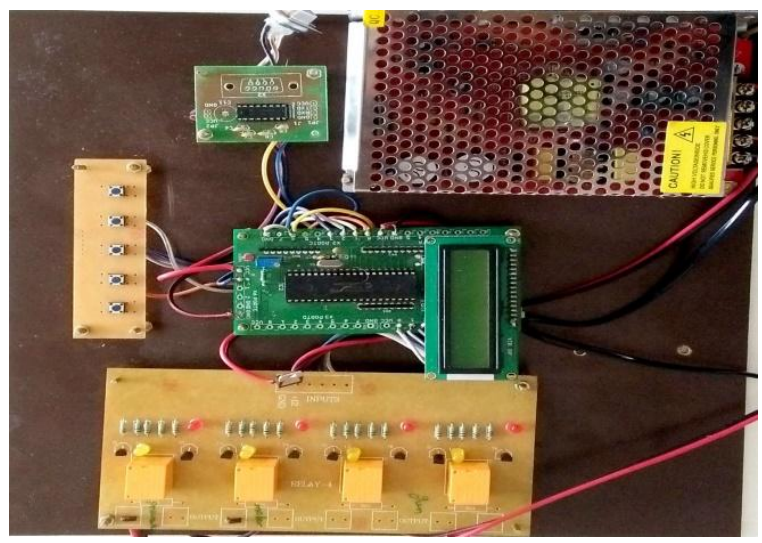

Fig 5 Circuit Diagram

Camera mounted above the conveyor belt captures the nuts images which is on moving conveyor belt. Using Mathematical morphology algorithm which detects the object of the captured image and extracts the feature (diameter) of the nut. It derives the values from the image using MATLAB and compare with stored area of the object. If any fault detected, through microcontroller the electric signals would be passed to the separator and separator will redirect the object to the secondary conveyor belt. In this way, faulty objects are identified and isolated from the conveyor belt.

\section{CONCULSION}

In this automated fault detection and identification system, image processing based solution was proposed and implemented to identify defected/faulty components passing through the conveyor belt. Diameter of the nuts are considered as ROI and extracted for experiment to identify the defected components. Proposed system is experimented for collection of components and we have obtained $85 \%$ of accuracy. Due to lightening conditions, image acquisition varies ,hence there is a discrepancy in end results .The same experiment can be carried for bolt and other mechanical objects with the minor modification in the code. Contour based feature extraction can be used to enhance the accuracy and it would be considered for future work

\section{REFERENCE}

1. Amol Dhenge,Pallavi Keskar,"Computer Vision Based Object Sorting \& Fault Detection Using Ann",IFERP,volume 1, Issue 5, April 2015

2. Dr. Jangala. Sasi Kiran, N. Vijaya Kumar "A Literature Survey on Digital Image Processing Techniques in Character Recognition of Indian Languages" International Journal of Computer Science and Information Technologies, Volume 6 , 2015.

3. LekhaBhausahebKachareME (E \& TC) “ Object Sorting Robot Using Image Processing “ ,International Journal of Electronics, Electrical and Computational System ,Volume 5,Issue 7, July 2016

4. B.Karunamoorthy, Dr.D.Somasundareswari, S.P.Sethu“Automated Patterned Fabric Fault Detection Using Image Processing Technique in Matlab" International Journal of Advanced Research in Computer Engineering \& Technology Volume 4 Issue 1,,January 2015

5. S Nakadate, T Yatagai,,H Saito "Electronic speckle pattern interferometry using digital image processing techniques", Applied Optics, vol. 19, Issue 11, 1980, pp. 1879-1883.

6. Salem Saleh Al-amri ,N.V. Kalyankar and Khamitkar S.D "Image Segmentation by Using Thershod Techniques" Journal of computing, vol. 2, Issue 5, May 2010, pp.83-86.

7. R. J. Beattie, "Edge detection for semantically based early visual processing," Ph.D. dissertation, Univ. Edinburgh, 1984.

8. C. Bingham, M. D. Godfrey, and J. W. Tukey, "Modem techniques of power spectrum estimation," IEEE Trans. Audio Electroacoust., vol. AU-15, no. 2, pp. 56-66, 1967.

9. R. A. Brooks, "Symbolic reasoning among 3-D models and 2D images," Dep. Comput. Sci., Stanford Univ., Stanford, CA, Rep. AIM343, 1981.

10. J. F. Canny, "Finding edges and lines in images," M.I.T. Artificial Intell. Lab., Cambridge, MA, Rep. Al-TR-720, 1983

11. Latha, L.,Thangasamy, S," Efficient method of person authentication based on fusion of best bits in left and right irises", 2012 International Journal of Biometrics

12. L Latha, S Thangasamy,"Providing multimodal biometric authentication using five competent traits", The Imaging Science Journal, 2013

13. $1 \mathrm{~K}$ Zuiderveld, "Contrast limited adaptive histogram equalization", In Graphicsgems IV. SanDiego,CA, USA: Academic Press Professional,Inc.; 1994, pp:474-485. 